Available online at GSC Online Press Directory

GSC Biological and Pharmaceutical Sciences

e-ISSN: 2581-3250, CODEN (USA): GBPSC2

Journal homepage: https://www.gsconlinepress.com/journals/gscbps

(RESEARCH ARTICLE)

\title{
Epigenetics, exposome and development of the intestinal microbiota in the neonate
}

\author{
Paola Sánchez Benitez ${ }^{1}$ and Rocío Pérez y Terrón ${ }^{2, *}$ \\ ${ }^{1}$ Faculty of Chemical Sciences Autonomous University of Puebla. Av. San Claudio and 18 Sur, Building 105-H, City \\ University C. P. 72570 Puebla, Mexico. \\ ${ }^{2}$ Microbiology and Molecular Biology Laboratory, Biological Sciences Faculty, Autonomous University of Puebla, Building \\ 112-A, City University C. P. 72570 Puebla, Mexico.
}

Publication history: Received on 10 November 2020; revised on 18 November 2020; accepted on 20 November 2020

Article DOI: https://doi.org/10.30574/gscbps.2020.13.2.0367

\begin{abstract}
The intestinal microbiota is currently known as a "metabolic organ" that significantly influences the health of the host from the first years of life, being a crucial factor for optimal development of immunity and regulation of different physiological processes such as digestion, absorption, metabolism and synthesis of nutrients. This work aims to show the relationship between epigenetics, the exposome and the development of the intestinal microbiota in the newborn according to the route of birth. A documentary review of the literature of the last 5 years was carried out and it was found that the majority of non-communicable diseases are due to epigenetic modifications that can occur in the prenatal stages, together with environmental factors that also contribute to these epigenetic changes, term known as an exposome. In this sense, the greatest exposure of microorganisms for the development of intestinal colonization is at the time of birth, being Bifidobacterium one of the most important genera that contribute to immune function, found to a lesser extent in newborns born by abdominal route (cesarean section), this type has been associated with dysbiosis of the intestinal microbiota, generating consequences in the development of diseases such as obesity, diabetes, asthma, food allergy and autism spectrum disorder. Allowing to conclude that both epigenetics and the exposome and the intestinal microbiota are simultaneously related from the early stages of life and can be the cause of various noncommunicable diseases.
\end{abstract}

Keywords: Epigenetics; Exposome; Microbiota; Neonate

\section{Introduction}

The period of life in which the epigenetic modifications of DNA are most intense goes from conception to 2 years of age, known as the "period of 1000 days", at this time early nutrition, psychic factors and the environment through epigenetic changes may play a key role in the programming of neonatal fetal development by influencing the susceptibility of the subsequent development of noncommunicable diseases [1]. The microbiota of the individual is determined from this moment on and predominantly during the first year of life, this will largely depend on the way in which birth occurs [2].

The microbiota is defined as the set of microorganisms that coexist with humans, occupying specific niches on the surfaces of the skin and mucosa, in a symbiotic relationship with the host [3]. The total number of microorganisms and their genetic material is defined as the microbiome. This term was coined in 2001 by Joshua Lederberg, an American molecular biologist who won the Nobel Prize in Medicine in 1958 [4].

\footnotetext{
* Corresponding author: Rocío Pérez-y-Terrón

Microbiology and Molecular Biology Laboratory, Biological Sciences Faculty, Autonomous University of Puebla, Building 112-A, City University C. P. 72570 Puebla, Mexico.
}

Copyright (C) 2020 Author(s) retain the copyright of this article. This article is published under the terms of the Creative Commons Attribution Liscense 4.0. 
The intestinal microbiota is heterogeneous and is composed mainly of bacteria, with a minority of viruses (intestinal viroma), fungi and yeasts $[5,6]$. The human intestine, primarily the large intestine, houses the highest amount of microbiota in the body compared to other niches such as the skin, vagina, mouth, and ears. It contains $10^{14}$ bacteria that represent 10 times the total number of human cells. In particular, after bacterial colonization in infants, the intestinal microbial composition is unique for each individual [7].

Today it is considered a "metabolic organ" [8], which is an important mediator in health and disease and interacts with various organs and systems in the body, including the brain, liver, skeletal and cardiovascular systems. Being a complex ecosystem shaped by evolution, with host-bacteria associations that promote a delicate balance, developed to modulate the immune response and promote health [9]. Through interactions with the host, the intestinal microbiota participates in the regulation of many physiological processes such as food digestion, nutrient absorption and metabolism, the synthesis of vitamins (K, B12, biotin, folic and pantothenic acid) and bile acids, as well as the modulation of innate immunity, promotion of maturation and integrity of the intestinal epithelium, growth and development of strains, up to the prevention of the spread of pathogenic microorganisms [6, 10, 11]. Multiple bacteria in the gut microbiota are involved in the production of a wide variety of enzymes, including cytochrome (CYP) P450, which carry out different types of metabolic reactions to a large number of oral foods and medications. These reactions include the processes of hydrolysis, decarboxylation, deamination, demethylation, ring opening of heterocyclic compounds, reduction, dehydroxylation, aromatization and dehalogenation [2].

Some evidence suggests that early events in fetal and neonatal life can program some diseases, which has demonstrated the importance of perinatal risk and suggests that the increase in the frequency of chronic diseases is caused by the combination of genetic and environmental factors in the first years of life [12]. The fetus was previously thought to develop under sterile conditions, but recent data have suggested the presence of a microbiota in the uterus, particularly in the placenta [13]. In addition to this, the neonatal and infantile periods have been shown to be important stages in the permanence of the intestinal microbial community [14]. The mode of birth affects the initial bacterial establishment, as the bacteria found in the vaginally delivered baby are very different from those experienced through cesarean deliveries [6], which can potentially affect the neonatal immune response [15]. It is now well known that elective caesarean section can affect the gut microbiota of the newborn up to six years of age [12].

According to the health statistics of the Organization for Economic Cooperation and Development (OECD), which is an international intergovernmental organization of which 37 member countries are members, "the rates of caesarean sections have increased in most of the countries of the OECD, averaging $20 \%$ in 2000 to $28 \%$ in 2013 . The increase has been particularly strong in middle-income countries such as Turkey, Mexico and Chile, where caesarean section rates now account for $45 \%$ or more than all deliveries" [16].

The World Health Organization recommends that the proportion of cesarean births should be between 10-15\%. In Mexico, the Official Mexican Standard 007 establishes as acceptable 15\% in second-level hospitals and 20\% in thirdlevel hospitals; however, the rate of cesarean sections according to the 2012 National Health and Nutrition Survey is $45.1 \%$ of all births $[17,18]$. The high frequency of caesarean sections is already considered a public health problem [19], in parallel to this increase, the rates of allergic diseases and childhood obesity have shown a sustained increase over time [3]. Due to the above, the objective of this review is to analyze the relationship between epigenetics, exposome and development of the intestinal microbiota in the newborn, according to the route of birth and its impact on long-term health.

\section{Methodology}

A documentary review of the literature was carried out on the current situation between epigenetics, exposome and intestinal microbiota, as well as its impact on the prenatal and postnatal stages. It was also related to the development of the intestinal microbiota in the full-term neonate, with its possible alterations according to the route of birth. This made it possible to take advantage of all the scientific information and infer about the consequences of dysbiosis caused by the abdominal birth route (cesarean section) in the newborn. Articles published in databases were selected, covering the period 2015-2020. 


\section{Results and discussion}

\subsection{Composition of the gut microbiota}

In its early days, the ecological dynamics of human commensal bacteria has been a field of difficult study, primarily because of the technical problems associated with the non-culturable nature of most of these bacteria. However, with the technological advance that has led to the emergence of next-generation sequencing techniques, in addition to complex bioinformatic platforms [20], it has been determined that the intestinal microbiota is made up of four bacterial phyla: Firmicutes and Actinobacteria (Gram positive) and, Bacteroidetes and Proteobacteria (Gram negative) [21]. The Firmicutes: Bacteroidetes ratio has been suggested as an indicator of the degree of maturation of the human intestinal microbiota, being lower in infants and the elderly compared to adult individuals [22].

Of the genes identified at the intestinal level, $98 \%$ are bacterial and between 1000 and 1150 bacterial species are described, with an average of 160 species per individual. As has been established with the development of the Human Microbiome Project, the diversity of the microbiome is unique for each individual and is strongly related to the conditions of the microbial habitat. At the intestinal level, the spectrum of the bacterial community varies in each segment of the intestine considered, from the cecum to the rectum [23].

\subsection{Relationship between epigenetics, exposome and gut microbiota}

Currently the interpretation of the correlation between epigenetics, exposome and the intestinal microbiota of an individual can contribute to a better understanding of the etiopathogenesis of some non-communicable diseases [24]. The origin of the development of health and disease highlights the importance of the period of the first 1000 days, a period that includes: 270 days from conception to birth, 365 days of the first year of life and 365 days of the second year of life [2]. During this time, the imprinting of epigenetic DNA is more active, which means that the genes "preserve the memory of where they come from" (father or mother), in addition to forming most of the organs, tissues, physical and intellectual potential and eating habits [1,24].

Changes in the quality or quantity of the nutrients consumed by the pregnant woman and the child can permanently influence their developing organs, the effects are known as "fetal programming" and constitute an important factor in the appearance of diseases non-communicable, mainly in adulthood. Thus, nutritional deficiencies during pregnancy that cause delayed intrauterine growth are associated with an increased risk of type 2 diabetes and coronary heart disease in the offspring [24].

Epigenetics refers to the set of functional elements that regulate the gene expression of a cell without altering the DNA sequence [25], it is also a field of study to understand how the origins of fetal programming are subject to the presence of certain environmental stimuli during pregnancy, the latter being able to promote variation in gene expression from the same fetal genome, resulting in different phenotypes [26]. Several studies have shown that epigenetic mechanisms (DNA methylation, histone modification, and the effect of small non-coding RNAs) are responsible for tissue-specific gene expression during embryonic, fetal, and neonatal growth. One of the most studied epigenetic mechanisms is DNA methylation, as it is a regulator of chromosomal processes that inactivate gene expression, today it can be stated that epigenetic modifications are potentially reversible and are influenced by the environment $[1,26,27]$. It is considered that epigenetic mechanisms can be activated by various factors during pregnancy due to an unfavorable intrauterine environment (Table 1). Epigenetic modifications present a high degree of stability, if genes linked to diseases are turned on or off, they can remain in a family for many generations (transgenerational transmission without altering the DNA sequence, due to the alteration of the epigenome) [1].

Table 1 Factors involved in the activation of epigenetic mechanisms during pregnancy

\begin{tabular}{|l|}
\hline Hypoxia \\
\hline Maternal violence \\
\hline Drug's use \\
\hline Stress \\
\hline Bad nutrition \\
\hline Maternal pathologies \\
\hline Exposure to endocrine disruptors \\
\hline Vulnerability in the social determinants of health \\
\hline
\end{tabular}

[1] 
Epigenetic mechanisms act in the development of complex non-communicable diseases such as cancer, asthma, arterial hypertension, cardiovascular disease, type 2 diabetes mellitus, epilepsy, Alzheimer's, psychiatric and immunological disorders and obesity. They are also involved in the cognitive process of memory and neuroplasticity, as well as in the expression of attitudes, psychopathies and personality [25, 28].

All environmental exposures during the life course (including lifestyle and other non-genetic factors), from the prenatal period onwards are known as exposomes, this concept was introduced for the first time in 2005 by Christopher P. Wild, a molecular epidemiologist and director of the International Agency for Research on Cancer in the United States. The exposome was developed to highlight the need for more accurate, reliable, and comprehensive environmental exposure data to complement the impressive advances made in measuring the human genome [29, 30]. The exposome contains three overlapping domains of non-genetic factors that contribute to disease risk: general external, specific external, and internal (Table 2) [2, 24].

Table 2 Factors Involved in the Domains Contained by the Exposome

\begin{tabular}{|l|l|l|}
\hline General external domain & Specific external domain & Internal domain \\
\hline Social capital & Occupation & Inflammation \\
\hline Education level & Lifestyle & Metabolism \\
\hline Climatic factors & Exposure to pollutants & Oxidative stress \\
\hline Rural or urban environment & - & Gut microbiota \\
\hline Socioeconomic level & - & Circulating hormones \\
\hline Residence location & - & - \\
\hline
\end{tabular}

The first 1000 days of life can be an important starting point for describing the dynamics of the exposome. The pregnancy period is important to develop a lifetime exposure, in addition to multiple environmental factors such as: environmental pollution, tobacco smoke, noise, pesticides and radiation, which can lead to serious and chronic diseases. These environmental exposures, during this stage, can permanently modify the body's structure, physiology, and metabolism, which have been associated with intrauterine growth retardation and neurotoxic, immunotoxic, and obesogenic effects in children [2, 29].

The presence of gestational diabetes or obesity in pregnant women favors an intrauterine environment of nutritional imbalance and excess energy in the transport of metabolic substrates through the placenta to the fetus. As a consequence, the offspring develop larger amounts of physiologically unnecessary adipose tissue and with this, they are more likely to subsequently develop metabolic disorders [26].

Recent studies have shown that certain prenatal exposures associated with childhood asthma are also related to changes in DNA methylation in the child's peripheral blood cells. Maternal smoking during pregnancy was shown to alter the infant's methyloma, implying that prenatal smoke exposure can lead to adverse fetal outcomes, including asthma [31]. Similarly, exposure to air pollution during embryonic development and early in life can result in epigenetic modification (methylation of DNA in the placenta) and reprogramming of the fetus, which can influence susceptibility to disease in the adulthood [32]. Furthermore, it is assumed that the infant's exposure to chemicals from human blood and milk may affect immune tolerance, intestinal colonization (microbiome), small bowel development, nutrient availability and absorption during prenatal periods and postnatal [24].

The gastrointestinal exposome is defined as the interaction between the host, the intestinal microbiota and all the environmental exposures to which the human being is subjected throughout life. Among the most representative components of this include: drugs, nutrients, pathogens transmitted by food, biological toxins and other synthetic contaminants. The gastrointestinal exposome can alter the composition, diversity and biological activity of the intestinal microbiota. For example, a high-fat diet can change the amount and diversity of the intestinal microbiota, which favors the decrease of Bacteroides and the increase of Firmicutes and Proteobacteria; while a high fiber diet increases the proportion of Firmicutes [2]. 


\subsection{Importance of the intestinal microbiota in the newborn}

The microbiota has a commensal relationship with the host and plays a key role in human health and disease. Therefore, the homeostatic balance of the gut microbiome is extremely beneficial to the host. The alteration of the microbiota is known as "dysbiosis", which has been related to 3 different phenomena, which can occur at the same time: 1) loss of beneficial organisms, 2) excessive growth of potentially harmful bacteria and 3) loss of general microbial diversity [10]. The assembly of the intestinal microbiota begins before and during the parturition process and evolves with feeding during infancy and other factors that are highly related (Fig. 1). The microbiome of a newborn cannot be fully understood in terms of the isolated individual, but in deep relation to the microbiome of its mother. Variation in the composition of the human intestinal microbiota is frequently attributed to increasing rates of cesarean deliveries, the use of antibiotics and feeding based on dairy formulas, among others [20].

In utero

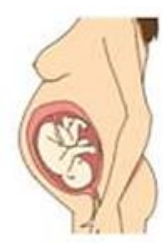

Birth

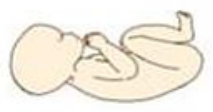

Infancy

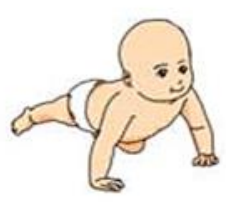

Childhood

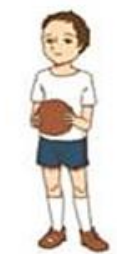

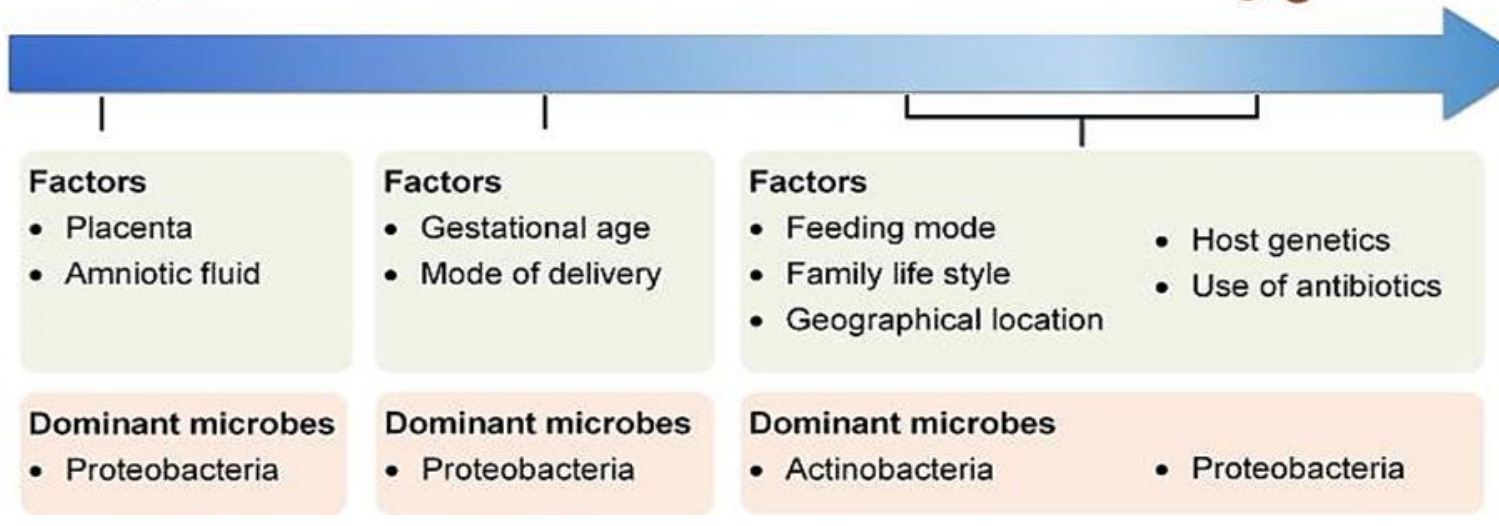

Figure 1 Factors involved in the development of the intestinal microbiota [14]

Studies have shown the importance of the microbiota during brain development, including the indirect effect of the microbiome on tryptophan metabolism and serotonin (5-HT) synthesis, 5-HT is known to be crucial for development of the Central Nervous System (CNS) [33], for this reason microbial colonization in the neonate coincides with key periods of neurological development. Some evidence suggests an association between the interruption of this colonization process and central nervous system dysfunction with the potential to cause adverse psychological outcomes. The reciprocal interaction between this system and the enteric nervous system through the signaling of various molecules produced by the microbiota and the brain can influence the intestine through neurotransmitters that have an impact on immune function and by variations in cortisol levels can influence intestinal motility and permeability [5].

\subsection{Development of the intestinal microbiota in the neonate}

For a century, the paradigm has been that the uterus is a sterile environment, being the first microbial exposure of the human being at the moment of birth. It's worth saying that most of the studies that established the sterile uterus paradigm date back to research using traditional culture-based methods and traditional microscopy, which are now considered deficient in assessing a microbiome. Therefore, researchers have resorted to the use of molecular techniques, which have made it possible to detect bacteria in placentas, amniotic fluid and meconium (a baby's first stool), in normal pregnancies (Fig. 2). In the amniotic fluid, a microbial community characterized by low diversity with a predominance of Proteobacteria has been found. Furthermore, it has been suggested that the uterus contains its own microbiome that may contribute to fetal colonization, as the placenta develops from fetal trophoblasts and the maternal decidua (the inner lining of the uterus), suggesting several pathways bacterial access to the placenta, including ascension from the lower genital tract, entry through the mother's bloodstream, active transport of microbes by immune cells from the intestine or oral cavity, and it has also been proposed that this fetal colonization could occur through ingestion of amniotic fluid containing bacteria (Fig. 3) [8,14,34,35]. 
Regarding the data suggesting that intrauterine bacteria interact with the developing fetus, these data have been provided by studies in germ-free animals, that is, animals lacking microbial colonization, in which it has been observed that the function immune at birth differs from that of conventional newborn animals [36].

In 2014, a team of researchers led by Dr. Aagaard identified bacterial deoxyribonucleic acid (DNA) in placental tissue. Until that time, the isolation of any type of bacteria in the placenta or amniotic fluid had the necessary consideration of intrauterine infection and, therefore, what was expected was the necessary identification of inflammatory processes associated with the increase in acute phase reactants and the coincident or later development of infection symptoms. The identification of a placental microbiome is not necessarily associated with clinical or laboratory signs of infection, and a normal term gestation sometimes develops. This would indicate that the neonatal microbiome could be seeded before birth, but regardless of whether or not the prenatal colonization of the fetus is performed as suggested, delivery marks the time of extensive exposure to microbial communities of fecal, vaginal, and skin and environmental origin, so this event has an important impact on colonization of the newborn intestine [23].

In relation to the data supporting the "Uterus colonization hypothesis", these should be taken with caution, since most of them were obtained with particular methodological limitations, for example, detectable bacterial DNA could belong to dead organisms this consideration being relevant for the placenta, since within its functions is the elimination of microorganisms that could be present in the blood; very few studies have found viable microorganisms in the fetal environment. In addition, it has been noted that highly sensitive molecular techniques used to study the microbiome related to the fetus tend to detect contaminating microbes, thus generating false positive results. Avoiding contamination is nearly impossible when collecting environment-related samples within the uterus of a clinical setting. Despite the above, the presence of microorganisms in meconium and amniotic fluid is frequently considered evidence that supports the hypothesis of colonization in the uterus [34, 35].

A

STERILE WOMB PARADIGM

Fetus and placenta are sterile.

The gut microbiome is acquired after birth.

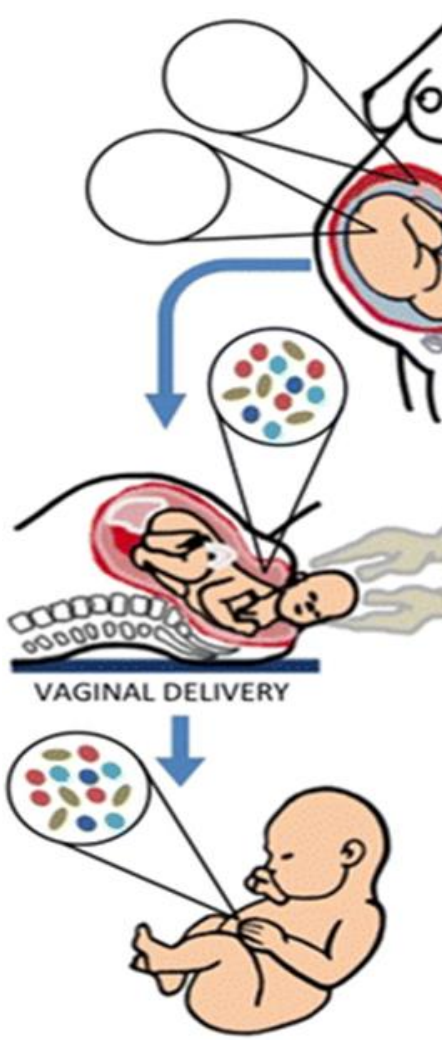

B IN UTERO COLONIZATION HYPOTHESIS

The placenta harbors its microbiome. Colonization of the gut begins in utero.

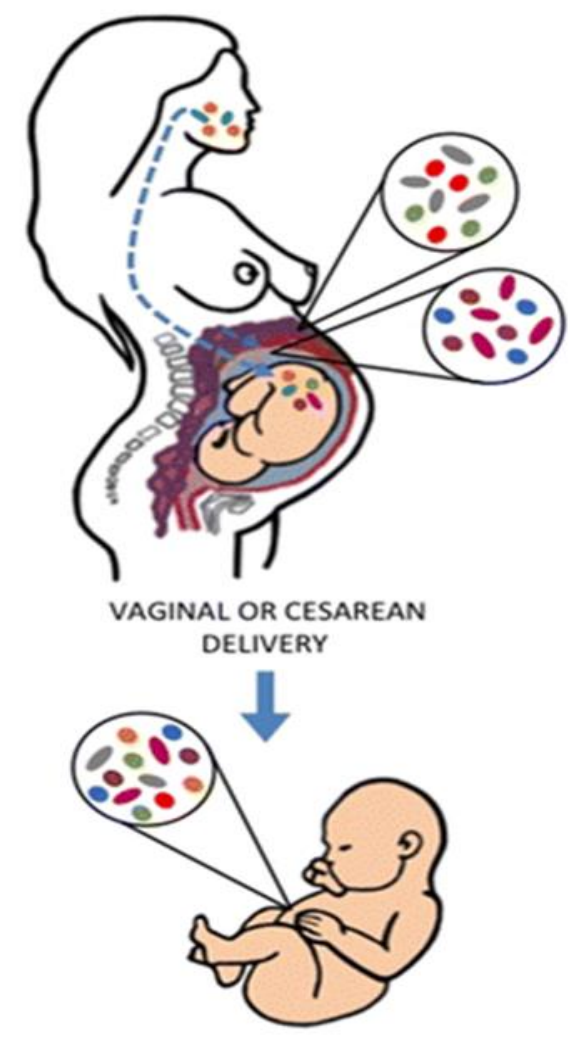

Figure 2 Schematic representation of the opposing concepts by which the human microbiota is acquired in early life A) Paradigm of the sterile uterus and B) Hypothesis of colonization in the uterus [35] 


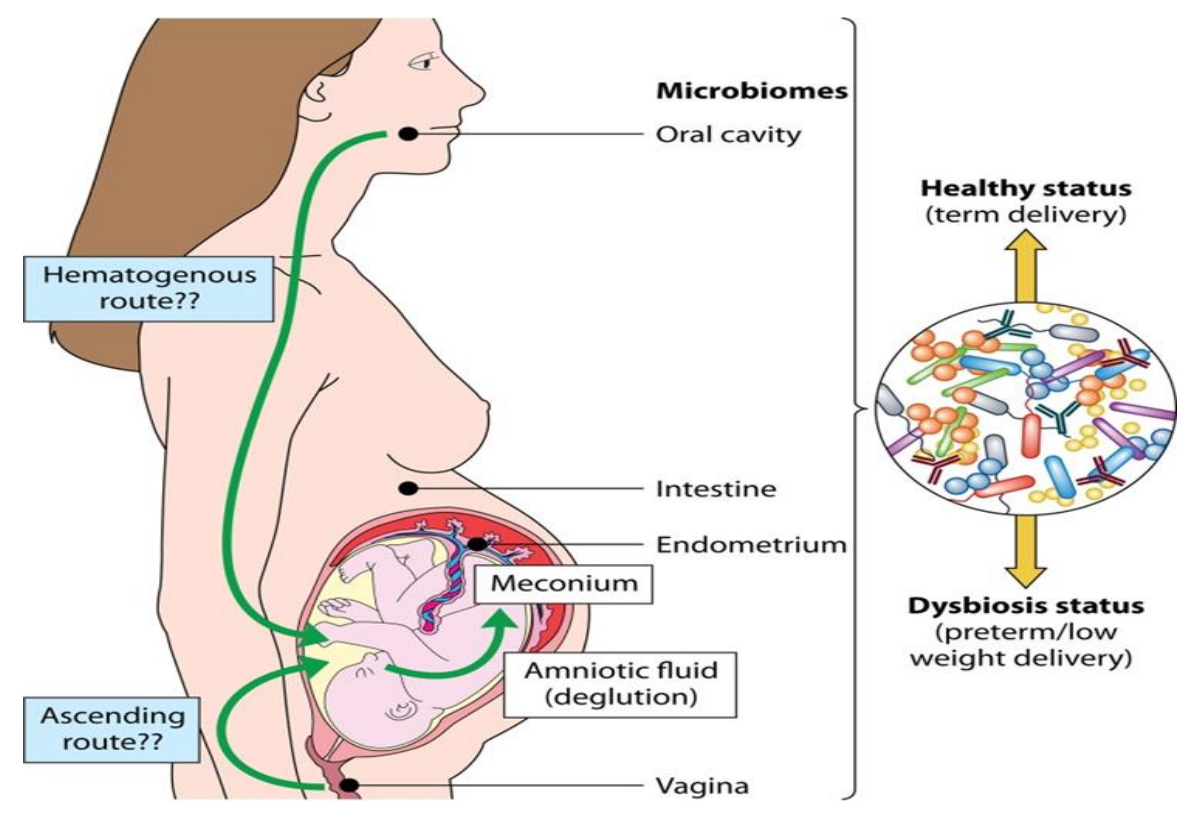

Figure 3 Colonization routes of maternal microbiomes towards the neonate [34]

\subsection{Gut microbiota according to the type of delivery (natural vs. cesarean section)}

In recent years, several studies have identified that the microbiome that initially colonizes the newborn differs significantly depending on whether the delivery was vaginal or by cesarean section [8]. Neonates born vaginally have a gut microbiota taxonomically similar to the mother's gut and vaginal microbiota. In contrast, in the intestinal microbiota of those born by caesarean section, microorganisms from the skin of the mother and the environment prevail, such as the oral microbiota and the skin of people who come into contact with the baby (Table 3) [20,21,23,37,38,39,40].

Table 3 Comparison of the microbiota in full-term infants

\begin{tabular}{|l|l|l|}
\hline Vaginal route & \multicolumn{2}{|l|}{ Abdominal route (caesarean section) } \\
\hline Greater prevalence & Greater prevalence & Less prevalence \\
\hline Bacteroides & Corynebacterium spp. & Actinobacterias \\
\hline Bifidobacterium & Propionibacterium spp. & Bacteroides \\
\hline Lactobacillus spp. & E. hormaechei & Bifidobacterium \\
\hline Parabacteroides & E. cancerogenus & \\
\hline Escherichia & E. faecalis & \\
\hline Prevotella spp. & E. faecium & \\
\hline Atopobium o Sneathea & E. cloacae & \\
\hline & H. parainfluenzae & \\
\hline & H. aegyptius & \\
\hline & H. influenzae & \\
\hline & H. haemolyticus & \\
\hline & S. saprophyticus & \\
\hline & S. lugdunensis & \\
\hline & S. aureus & \\
\hline & S. australis & \\
\hline & V. dispar & \\
\hline & V.párvula & \\
\hline & K. pneumoniae & \\
\hline & K. oxytoca & \\
\hline & C. perfringens & \\
\hline & &
\end{tabular}

$[37,21,38,39,20,23]$

The great abundance of Bifidobacterium species in babies is considered to promote the maturation of the healthy immune system, at the same time it has evolved to selectively digest special sugars in breast milk and metabolize sugars, 
producing various microbial fermentation products, such as acetate of short chain fatty acids, which lowers the pH, creating an acidic intestinal environment, while also metabolizing amino acids from breast milk into aromatic lactic acid, which has emergent functions that include improving the integrity of the infant's intestinal wall. The decrease in the number of intestinal Bifidobacteria has clinical relevance, since this type of intestinal dysbiosis early in life predisposes to inflammation. On the other hand, the high presence of Clostridium difficile is considered one of the main in-hospital risks of severe gastrointestinal infections and asthma during childhood, associating it with a higher risk in children born by cesarean section, since they show a significantly higher percentage of colonization of this microorganism $[41,42,43,44]$.

Furthermore, an additional factor that must be considered in establishing the newborn's microbiota is gestational age [20]. Gestational age is the time elapsed from the first day of the mother's last menstruation to the birth of her child. The term gestation is 40 weeks, however, those born between 37 and 41 weeks of gestation are considered within this category [45]. In fact, preterm and term infants show significant differences in the composition of their microbiome [20]. The intestinal microbiota of preterm infants is characterized by types of potentially pathogenic bacteria that are commonly found in the hospital environment, this can be reflected in the feces with higher levels of Enterobacteriaceae, Enterococcus and other pathogenic (opportunistic) microorganisms, compared to the fecal material from term-gestated newborns, lastly preterm infants exhibit late intestinal colonization of Bifidobacterium or Bacteroides [34,41].

If the number of intestinal bacteria per gram of stool at one month of age were counted, the result would be significantly higher in newborns vaginally [23]. Actinobacteria have been reported to be present in the feces of babies delivered by caesarean section on days 7 to 15 after birth [34]. In this way, differences in the gut microbiome of caesarean section newborns may be visible up to seven years after birth [42]. In addition, the differences between the intestinal microbiota of babies born vaginally and caesarean section, it has been reported that the latter have reduced levels of immune mediators such as cytokines and an increase in neonatal respiratory distress, or even mortality has been observed and medium and long-term disability [37].

\subsection{Consequences of intestinal microbiota dysbiosis and its association with the abdominal birth route (cesarean section)}

The neonatal gut microbiota is a complex bacterial ecosystem linked to nutrition, metabolism, and stimulation of the immune system that can affect the optimal development of the child during different stages of growth [36]. There are several perinatal factors, such as cesarean delivery, type of feeding, antibiotic treatment, gestational age or environment that can affect the pattern of bacterial colonization and cause dysbiosis (Fig. 1) [13].

Caesarean section has saved an extremely high number of lives; however, in recent decades, it has become standard practice and sometimes unnecessary or worse, convenient. In the last 2 decades, caesarean section rates have approached 50\% in some countries, showing little interest in how the "mode of delivery" can affect long-term health in the newborn and into infancy [9]. This early dysbiosis can persist for several months and therefore can have long-lasting functional effects with an impact on disease risk later in life [13]. In recent years, an increased risk of food allergies, asthma, obesity, type 1 diabetes, autism spectrum disorder has been reported as more frequent etiologies in infants after caesarean section than after vaginal delivery $[7,9,46]$.

\subsubsection{Allergies and asthma}

A drastic increase in the incidence of allergic diseases and asthma has been observed in industrialized countries, partly associated with industrialization itself, but it has also been related to a reduction in exposure to microorganisms during the neonatal period and in the first years of life, in this sense, babies at risk of asthma have a reduced amount of certain taxa of bacteria such as Faecalibacterium and Bifidobacterium [4, 47]. Allergic disorders have been associated with alteration in the microbiota [11], children born by cesarean section show an imbalance between Th1 and Th2 lymphocytes, presenting a significantly lower level of Th1 response and an increase in Th2, which is associated with an increased risk of developing allergic diseases and asthma [42,48]. In one study, a cohort of 219 children showed an increased risk of asthma at 7 years of age in the group of newborns by caesarean section as opposed to those born by vaginal delivery [3].

\subsubsection{Food allergy}

It is defined as an adverse immune response to a food and can be mediated by immunoglobulin E (IgE) (eg, Urticaria and anaphylaxis) or not mediated by IgE (eg, induced enterocolitis syndrome due to dietary proteins and eosinophilic esophagitis). IgE-mediated food allergy has been associated with immune dysregulation and impaired intestinal epithelial integrity, thus there is interest in a possible link to the gut microbiome. Studies evaluating the profile of the 
gut microbiome have revealed important microbial differences in patients with food allergies compared to healthy patients, similarly other studies have provided evidence that microbiome dysbiosis precedes the development of food allergies [49].

\subsubsection{Obesity}

Worldwide, it is presented as an epidemic behavior. In the study of the origin of this disease, currently not only is the genetic factor related as a determinant of obesity, but research is pointing to the intestinal microbiota as a factor that influences the development of this pathology. Recent studies suggest that both Bifidobacteria and Bacteroides appear to have a protective effect against obesity, which translates into an increased risk for children born by cesarean section [11]. A first epidemiological study in a cohort of 1255 newborns showed that newborns by caesarean section were twice as likely to be childhood obesity, even after adjusting for maternal body mass index, birth weight, and other confounding variables [13].

\subsubsection{Type 1 diabetes (T1D)}

According to the most recent estimates from the International Diabetes Federation, in 2017, the number of young people ( $<20$ years) worldwide with T1D was 1106500,13 times the number of the year 2015. T1D is believed to be mediated by infiltration and destruction of pancreatic $\beta$ cells by autoreactive $\mathrm{T}$ lymphocytes, the disease process often begins during neonatal life and is diagnosed mainly during childhood and adolescence, when cesarean delivery has been associated with an increased risk of T1D in infants [22].

\subsubsection{Autism spectrum disorder}

The etiology of autism is not well understood, but in recent years it has been proposed that the gut microbiota could play an important role in its development. Children with autism have been shown to have dysbiotic fecal microbiota, with a higher abundance of Bacteroidetes and a lower abundance of Firmicutes compared to controls [33]. In addition, there are studies that have reported poorer infant cognitive development and higher rates of autism spectrum disorders associated with cesarean delivery [46].

Berrocal et al, [28] mention that at the end of World War II between the years 1944-1945, a population in the west of the Netherlands was subjected to a state of prolonged fasting or starvation, which resulted in a condition of poor nutrition with a low protein and caloric load, this generated an effect on the epigenome of mothers who were in the gestation period. The women who managed to survive and later gave birth, it was observed that the children had a predisposition to suffer type 2 diabetes mellitus, obesity and cardiovascular diseases, due to the poor nutritional status of the mothers during pregnancy. The DNA methylation pattern is one of the main mechanisms to explain what happened in this population, since its alteration is related to the appearance of diseases due to changes in gene expression.

In this same sense, Lugo \& Martínez [50], refer that the nutritional status of the mother during pregnancy can influence embryological development and modulate its phenotype, without affecting the normal nucleotide sequence, through different epigenetic mechanisms, thus the availability of different micronutrients can result in the alteration of DNA methylation and the modification of histones, causing dysregulation in the expression of genes that program body development. According to the foregoing, it can be said that epigenetics and the environment influence simultaneously from the prenatal stages together with the mother, intervening in the quality of life of the individual.

\section{Conclusion}

From the moment of conception to birth, the individual is already predisposed to develop non-communicable diseases, due to the epigenetics and exposome of their predecessors. In the prenatal stage, the development of the intestinal microbiota occurs; In neonates born by caesarean section, it can cause dysbiosis, causing an impact on the health of the host and consequently an increased risk of suffering from food allergies, asthma, obesity, type 1 diabetes and autism spectrum disorder.

\section{Compliance with ethical standards}

\section{Acknowledgments}

The support to the Inter-Institutional Program for Strengthening Research and Postgraduate Studies in the Pacific DELFÍN and the Autonomous University of Puebla. 


\section{Disclosure of conflict of interest}

The authors declare that they have no conflicts of interest.

\section{References}

[1] Keklikián RI, Coronel AV. Microbiome and epigenetics in perinatology. 1000 days that can change destinies. Clínicas Perinatológicas Argentinas. ASAPER. 2018; 140-178.

[2] Cuadros CA, Ignorosa KR, Zárate FE, Toro E, Cervantes R, Montijo E, Cadena J, Serrano A, Ramírez JA. The exposome influence in the first 1,000 days old and gastrointestinal Health. Acta Pediatr Mex. 2018; 39(3): 265277.

[3] Harris PR. What do we know about the importance of the intestinal microbiota throughout life? Gastroenterol. Latinoam. 2019; 30(1): 13- 17.

[4] Pacheco Y, Bello Z, Góngora K. Human microbiome, implications in the health-disease process. Rev. electron. Zoilo. 2018; 44(1): 1-9.

[5] Michel ARJ, Izeta GAC, Torres AG, Michel ACMI. The microbiota and the human gut microbiome. (Between the keys to the kingdom and a new Pandora's box).Rev Sanid Milit Mex. 2017; 71(5): 443-448.

[6] Tinahones F. The importance of the microbiota in obesity. Rev Esp Endocrinol Pediatr. 2017; 8(1): 16-20.

[7] Rutayisire E, Huang K, Liu Y, Tao F. The mode of delivery affects the diversity and colonization pattern of the gut microbiota during the first year of infants' life: a systematic review. BMC Gastroenterol.2016; 16(86): 1-12.

[8] Torrachi A, Arcos M, Ochoa S, Mora M, Radax J, Palacios M, Salgado C. Bibliographic Review: The Human Microbiome. Rev Med HJCA. 2017; 9(3): 275-279.

[9] Francavilla R, Cristofori F, Tripaldi ME, Indrio F. Intervention for Dysbiosis in Children Born by C-Section. Ann Nutr Metab. 2018; 73 (3): 33-39.

[10] Sierra A, López O, Boj Ji, Milagro FI, Martínez A. Diet, Gut Microbiota, and Obesity: Links with Host Genetics and Epigenetics and Potential Applications. Advances in Nutrition. 2019; 10(1): 17-30.

[11] Zamudio VP, Ramírez JA, Toro EM, Cervantes R, Zárate F, Montijo E, Cervantes R, Zarate F, Montijo E, Cadena JF, Cázares JM. Importance of the gastrointestinal microbiota in pediatrics. Acta Pediatr Mex. 2017; 38(1): 49-62.

[12] Mancilla J. The birth path as a disease programmer. Rev Mex Pediatr. 2015; 82(4): 121-123.

[13] Butel M, Waligora A, Wydau S. The developing gut microbiota and its consequences for health. J Dev Orig Health Dis. 2018; 9(6): 590-597.

[14] Zhuang L, Chen H, Zhang S, Zhuang J, Li Q, Feng Z. Intestinal Microbiota in Early Life and Its Implications on Childhood Health. Genomics, proteomics \& bioinformatics. 2019; 17(1): 13-25.

[15] Sadler, Michelle. The Effects of Cesarean Section on Child Health: An Urgent Matter. Rev Chil Pediatr. 2018; 89(4): 561-562.

[16] OCDE Health Statistics. Why so much variation in caesarean section rates in OECD countries? 2020 [cited 2020 August 05]. Available from: http://www.oecd.org/els/health-systems/graph-of-the-month.htm

[17] Alonso E. M. G. Evolution of birth by cesarean section: The case of Mexico. DILEMATA 2015; (18): 27-43.

[18] Sepúlveda DL, Galván M, Soto GA, Méndez, DH. Factors associated with successful delivery of women with a history of cesarean section. Ginecol Obstet Mex. 2015; 83(12): 743-749.

[19] Bernal C, Nahín C. Cesarean section: current situation and associated factors in Mexico. Salud Quintana Roo. 2018; 11(40): 28-33.

[20] Serrano CA, León MA, Harris PR. Development of the gastrointestinal microbiota in infants and its role in health and disease. ARS MEDICA Revista De Ciencias Médicas. 2016; 41(1): 35-43.

[21] Calderón X, Guerrero A. Generalities in intestinal microbiota. Acta Científica de la Sociedad Venezolana de Bioanalistas Especialistas. 2019; 2(1): 27-34.

[22] Abdellatif AM, Sarvetnick NE. Current understanding of the role of gut dysbiosis in type 1 diabetes. Journal of Diabetes. 2019; 11 (8): 632-644. 
[23] Uberos J. Perinatal microbiota: Review of its importance in newborn health. Arch Argent Pediatr. 2020; 118(3): 265-270.

[24] Mena VR, Fernández BDLP. Relationship between intestinal microbiota, epigenetics and exposome in maternal and child health. Rev Cubana de Pediatr. 2019; 91(2): 1-13.

[25] Luna EJ, Castro M, León D. Epigenetics in the course of life: a challenge in the continuous training of health personnel. Rev Med Electrón. 2020; 42(1): 1669-1673.

[26] Ortega F, Chavira E, Piña G, Bárbara P. Adverse environmental factors during pregnancy: fetal epigenetic adjustments and their consequences on the phenotype. Mens. Bioquim. 2017; 41: 29-36.

[27] Castro JA, Krause BJ, Uauy R, Casanello P. Epigenetics in allergic diseases and asthma. Rev Chil de Pediatr. 2016; 87(2): 88-95.

[28] Berrocal JC, Gamez GD, Martínez LB, Redondo NG, Diazgranados IL, Acosta JI. Epigenetics and chronic noncommunicable diseases. Archivos de medicina. 2019; 15(4): 1-8.

[29] Santos S, Maitre L, Warembourg C, Agier L, Richiardi L, Basagaña X, Vrijheid M. Applying the exposome concept in birth cohort research: a review of statistical approaches. Eur J Epidemiol. 2020; 35(3): 193-204.

[30] Vicente MT, Ramírez MV, Capdevila LM, Terradillos MJ, López AA, Jiménez E, Reinoso L. Exposoma: a new concept in Occupational Health and Public Health. Rev Asoc Esp Espec Med Trab. 2016; 25(3): 176-183.

[31] DeVries A, Vercelli D. The neonatal methylome as a gatekeeper in the trajectory to childhood asthma. Epigenomics. 2017; 9(4): 585-593.

[32] Maghbooli Z, Hossein A, Adabi E, Asadollah E, Sadeghi M, Mohammad S, Zakeri L, Malek A, Radmehr M, Faghihi F, Aghaei A, Omidifar A, Aghababei Y, Behzadi H. Air pollution during pregnancy and placental adaptation in the levels of global DNA methylation. PLoS One. 2018; 13(7): 1-14.

[33] Ihekweazu FD, Versalovic J. Development of the Pediatric Gut Microbiome: Impact on Health and Disease. A.m. J. Med. Sci. 2018; 356(5): 413-423.

[34] Milani C, Durant S, Bottacini F, Casey E, Turroni F, Mahony J, Belzer C, Delgado S, Arboleya S, Mancabelli L, Lugli GA, Rodríguez JM, Bode L, de Vos W, Gueimonde M, Margolles A, van Sinderen D, Ventura M. The First Microbial Colonizers of the Human Gut: Composition, Activities, and Health Implications of the Infant Gut Microbiota. Microbiology and Molecular Biology Reviews: MMBR. 2017; 81(4): 1-67.

[35] Pérez ME, Arrieta MC, Ramer AE, Walter J. A critical assessment of the "sterile womb" and "in utero colonization" hypotheses: implications for research on the pioneer infant microbiome. Microbiome. 2017; 5(48): 1-19.

[36] Moreno JM, Collado MC, Larqué Elvira, Leis MR, Sáenz M, Moreno LA. The first 1000 days: an opportunity to reduce the burden of noncommunicable diseases. Nutr Hosp. 2019; 36(1): 218-232.

[37] Arboleya S, Suárez M, Fernández N, Mantecón L, Solís G, Gueimonde M, Reyes CG. C-section and the Neonatal Gut Microbiome Acquisition: Consequences for Future Health. Ann Nutr Metab. 2018; 73(3): 17-23.

[38] Carrasco IR, Lozano JC, Castañeda PC. Cesarean section as a conditioning factor for constipation, dermatitis and allergies in children of two units of the Mexican Institute of Social Security. Alergia, Asma e Inmunología Pediátricas. 2015; 24(1): 5-11.

[39] Navia LA, Ignorosa KR, Zárate FE, Bustamante R, Monjaraz EM, León JF, Mayans J. Gastrointestinal microbiota and its relationship with allergy. Acta Pediatr Mex. 2020; 41 (3): 135-147.

[40] Shao Y, Forster SC, Tsaliki E, Vervier K, Strang A, Simpson N, Kumar N, Stares M, Rodger A, Brocklehurst P, Field N, Lawley T. Stunted microbiota and opportunistic pathogen colonization in caesarean-section birth. Nature. 2019; 574(7776): 117-121.

[41] Dalby MJ, Hall LJ. Recent advances in understanding the neonatal microbiome. F1000 Research. Faculty Rev. 2020; 9(422): 1-8.

[42] Jagodzinski A, Zielinska E, Laczmanski L, Hirnle L. The early years of life. Are they influenced by our microbiome? Ginekologia polska. 2019; 90(4): 228-232.

[43] Mesa MD, Loureiro B, Iglesia I, Fernández S, Llurba E, García O, Solana MJ, Cabero MJ, Sainz T, Martínez L, Escuder D, Parra A, Sánchez M, Rodríguez G, Gómez D, Pérez M, Andreu V, Clotet J, Sailer S, Iglesias I, Cabañas F. The Evolving Microbiome from Pregnancy to Early Infancy: A Comprehensive. Review Nutrients. 2020; 12(133): 221. 
[44] Tribe RM, Taylor PD, Kelly NM, Rees D, Sandall J, Kennedy HP. Parturition and the perinatal period:can mode of delivery impact on the future health of the neonate? J Physiol. 2018; 596(23): 5709-5722.

[45] León LM, Gómez MF, Jiménez YC, Yenesis C, González MC, Moreno EA, Suárez E, Hernández AE, Mandujano JI, Payan T, Contreras MR, Hernández M, Guzmán LA y Hernández RA. Profile of the healthy term newborn in a private institution in Western Mexico. Rev. Med MD. 2016; 7(4): 275-279.

[46] Zhang T, Sidorchuk A, Sevilla L, Vilaplana A, Chang Z, Larsson H, Mataix D, Fernández L. Association of Cesarean Delivery With Risk of Neurodevelopmental and Psychiatric Disorders in the Offspring: A Systematic Review and Meta-analysis. JAMA network open. 2019; 2(8): 1-19.

[47] Peroni DG, Nuzzi G, Trambusti I, Di Cicco ME, Comberiati P. Microbiome Composition and Its Impact on the Development of Allergic Diseases. Frontiers in immunology. 2020; 11(700): 1-8.

[48] Montealegre SMF, Muñoz MFM, Vargas JFV. Cesarean delivery as a risk factor for the development of asthma in childhood. Rev Med Sinergia. 2019; 4(9): 3-3.

[49] Nance CL, Deniskin R, Diaz VC, Paul M, Anvari S, Anagnostou A. The Role of the Microbiome in Food Allergy: A Review. Children (Basel). 2020; 7(50): 1-18.

[50] Lugo NT, Martínez MH. Epigenetic mechanisms and the Notch signaling pathway at the origin of different congenital defects. Medicentro. 2018; 22(3): 197-207. 